\title{
Respiratory syncytial virus activates epidermal growth factor receptor to suppress interferon regulatory factor 1-dependent interferon-lambda and antiviral defense in airway epithelium
}

A Kalinowski ${ }^{1}$, BT Galen ${ }^{1}$, IF Ueki ${ }^{2}$, Y Sun $^{1}$, A Mulenos $^{1}$, A Osafo-Addo ${ }^{1}$, B Clark $^{1}$, J Joerns ${ }^{1}$, W Liu ${ }^{1}$, JA Nadel ${ }^{2}$, CS Dela Cruz ${ }^{1,3}$ and JL Koff ${ }^{1}$

Respiratory syncytial virus (RSV) persists as a significant human pathogen that continues to contribute to morbidity and mortality. In children, RSV is the leading cause of lower respiratory tract infections, and in adults RSV causes pneumonia and contributes to exacerbations of chronic lung diseases. RSV induces airway epithelial inflammation by activation of the epidermal growth factor receptor (EGFR), a tyrosine kinase receptor. Recently, EGFR inhibition was shown to decrease RSV infection, but the mechanism(s) for this effect are not known. Interferon (IFN) signaling is critical for innate antiviral responses, and recent experiments have implicated IFN- $\lambda$ (lambda), a type III IFN, as the most significant IFN for mucosal antiviral immune responses to RSV infection. However, a role for RSV-induced EGFR activation to suppress airway epithelial antiviral immunity has not been explored. Here, we show that RSV-induced EGFR activation suppresses IFN regulatory factor (IRF) 1 -induced IFN- $\lambda$ production and increased viral infection, and we implicate RSV F protein to mediate this effect. EGFR inhibition, during viral infection, augmented IRF1, IFN- $\lambda$, and decreased RSV titers. These results suggest a mechanism for EGFR inhibition to suppress RSV by activation of endogenous epithelial antiviral defenses, which may be a potential target for novel therapeutics.

\section{INTRODUCTION}

Respiratory syncytial virus (RSV), a member of the Paramyxoviridae family, persists as a significant human pathogen. It is reported that nearly all children worldwide will be infected with RSV, which is a leading cause of lower respiratory tract infections that contribute to significant morbidity and mortality. ${ }^{1}$ This exposure to RSV is associated with a subsequent risk of respiratory symptoms and asthma later in life. ${ }^{2}$ Similar to influenza virus, adaptive immune responses to RSV do not provide effective immune memory, ${ }^{3}$ which results in infection throughout life. In adults, RSV may cause pneumonia, but possibly more significantly, contributes to exacerbations of underlying chronic lung diseases (e.g., asthma, cystic fibrosis, and chronic obstructive lung disease (COPD)). ${ }^{2}$ Currently, a RSV-specific monoclonal antibody, Palivizumab, is recommended for the prevention of RSV infection in a small fraction of infants (e.g., born premature, or who have serious underlying disease). ${ }^{4}$ However, despite substantial disease burden, and significant efforts to identify a vaccine or antiviral therapy, no effective therapy is approved for RSV.

Airway epithelial cells (AECs) are the primary cell type for RSV infection, which induces inflammation and cell damage. RSV activates epidermal growth factor receptor (EGFR); (ErbB1/HER1), a tyrosine kinase receptor present in epithelial cells, which results in inflammation and mucin production. ${ }^{5-7}$ Although it was recently shown that inhibition of EGFR results in decreased RSV titers, ${ }^{7}$ a mechanism for this effect is unknown. We and others have shown that virus-induced EGFR activation suppresses antiviral innate immune responses. ${ }^{6,8,9}$ For example, DEXD/H box RNA helicase, DDX60, is an

${ }^{1}$ Section of Pulmonary, Critical Care, \& Sleep Medicine, Yale University, New Haven, Connecticut, USA. ${ }^{2}$ Division of Pulmonary, Critical Care, Allergy \& Sleep Medicine, University of California San Francisco, San Francisco, California, USA and ${ }^{3}$ Department of Microbial Pathogenesis, Yale University, New Haven, Connecticut, USA. Correspondence: JL Koff (jon.koff@yale.edu) 
interferon (IFN)-inducible cytoplasmic helicase that was found to be upstream of RIG-I and MDA-5 signaling. In models of vesticular stomatitis virus, sendai virus, and Hepatitis $C$ virus (HCV) infection, virus-induced EGFR activation suppressed DDX60-induced type I IFN production (e.g., IFN- $\alpha$ and $-\beta$ ). ${ }^{9}$

IFN signaling is a critical innate antiviral host response. Recent experiments suggest that IFN- $\lambda$ (lambda), a type III IFN, is the most significant IFN in AECs. ${ }^{10,11}$ Studies have shown that IFN- $\lambda$ is the primary IFN that regulates mucosal responses to viral infection, whereas type I IFNs are essential for clearance of systemic infection. ${ }^{11,12}$ In in vitro and in vivo experiments, we found that Influenza A virus and rhinovirus activate EGFR to suppress IFN lambda $(-\lambda)$ production. ${ }^{6}$ In addition, subsequent experiments showed that Influenza A virus, rhinovirus, and RSV activate EGFR to suppresses CXCL10 production, which contributes to recruitment of lymphocytes to target and kill virus-infected cells.

In response to RSV infection, Okabayashi et al., ${ }^{13}$ showed that IFN- $\lambda$, not type I IFNs, are the primary IFN produced by nasal epithelium. In addition, RSV was found to suppress IFN$\lambda$ in lung epithilium, ${ }^{14}$ which indicates its important role in antiviral immunity to RSV. The observation that IFN- $\lambda$ has a role in epithelial antiviral immunity has led to experiments to investigate the IRFs involved in epithelial IFN- $\lambda$ production. In airway epithelium, RSV activates IRF1. ${ }^{6,15-17}$ Subsequently, our laboratory and other investigators have shown that RSV activates IRF1 to induce IFN- $\lambda$ production. ${ }^{6,18}$ However, the potential for EGFR signaling to modulate IRF1 and IFN- $\lambda$ during RSV infection has not been explored. Here, we examined the effect of EGFR signaling on RSV infection and IRF1-dependent IFN- $\lambda$ production in airway epithelium.

\section{MATERIALS AND METHODS}

Reagents. EGFR tyrosine kinase inhibitor (AG 1478), epidermal growth factor (EGF), and transforming growth factor (TGF)- $\alpha$, diphenyleneiodonium chloride (DPI), neutralizing anti-EGFR (Ab-5) mAb, and an isotype-matched Ab were obtained from EMD Millipore (Billerica, MA, USA). Platelet-derived growth factor (PDGF) receptor tyrosine kinase inhibitor (AG 1295), Janus kinase (Jak) 1 inhibitor, IFN lambda $(-\lambda)$ polyclonal Abs, interferon $\lambda \mathrm{R}(\mathrm{IFN}-\lambda)(\mathrm{IL}-28 \mathrm{R} / \mathrm{IL}-10 \mathrm{R} \beta) \mathrm{Abs}$, and isotypematched Abs were purchased from Santa Cruz Biotechnology (Santa Cruz, CA, USA). $N$-propyl gallete (nPG) was purchased from Sigma (St. Louis, MO, USA). Gefitinib was purchased from Tocris Biosciences (Bristol, UK). RSV F protein was obtained from Sino Biological (Beijing, China).

Virus. RSV long strain was generously provided by Dr David Schnurr (California Department of Public Health, Viral \& Rickettsial Disease Laboratory). Virus was sucrose gradient purified as described previously, ${ }^{6,8}$ and was used in experiments for Figures 1 and 2. Figures 3 and 4 used unpurified RSV after subsequent experiments showed comparative levels of EGFR phosphorylation (EGFR-p) and cytokine production between purified and unpurified RSV (data not shown). RSV titers were determined by TCID50\% and plaque assay using $\mathrm{HeLa}$ and Vero cells purchased from American Type Culture Collection (Manassas, VA, USA), as previously described. ${ }^{6,19}$ RSV was UVinactivated by UV irradiation (UV RSV; GS Gene Linker; Bio-Rad, Hercules, CA, USA), and virus inactivation was confirmed by plaque assay. Cell culture supernatants from HeLa and Vero cells that were mock infected did not induce IFN- $\lambda$ in BEAS- $2 b$ cells above amounts induced by serum-free medium alone (data not shown).
Cell culture. Dr Walter Finkbeiner (San Francisco, CA, USA) generously provided primary human bronchial epithelial (NHBE) cells from healthy donors. NHBE cells were grown in bronchial epithelial growth medium (Lonza Walkersville, MD, USA) supplemented with growth factors, penicillin $\left(100 \mathrm{U} \mathrm{ml}^{-1}\right)$, and streptomycin $\left(100 \mu \mathrm{g} \mathrm{ml}^{-1}\right)$. Sixteen hours prior to viral infection, EGF and hydrocortisone were removed from cell culture medium, as previously described. ${ }^{6,8}$ Dr John Fahy (San Francisco, CA, USA) generously provided bronchial epithelial (BEAS-2b) cells, which are cultured in Rosewell Park Memorial Institute medium with $10 \%$ fetal bovine serum (Sigma-Aldrich). All cells were cultured at $37^{\circ} \mathrm{C}$ and $5 \% \mathrm{CO}_{2}$ as previously described. ${ }^{6,8}$

RSV was used at multiplicity of infection (MOI) of 0.1 in BEAS-2b cells, and at an MOI of 1.0 in NHBE cells based upon prior doseresponse experiments, as previously described. ${ }^{6} \mathrm{UV}$ RSV was used at MOI of 1 based upon RSV titers. Chemical inhibitors were added to cell cultures at the time of viral infection. AG 1478 and Gefitinib were used at $10 \mu \mathrm{m}$ because experiments have shown this concentration inhibits virus-induced inflammation. ${ }^{20-22}$ We confirmed that AG 1478 and Gefitinib $(10 \mu \mathrm{m})$ inhibited RSV-induced CXCL8 production in BEAS-2b cells, ${ }^{6}$ and neither inhibitor-induced cell toxicity as measured by $\mathrm{LDH}$ production at this concentration. ${ }^{6,8}$ In addition, we used EGFR small interfering RNA (siRNA) to confirm selectivity for EGFR. For experiments using the EGFR ligands EGF and TGF- $\alpha$, we used $10 \mathrm{ng} \mathrm{ml}^{-1}$ because we, and other investigators, have shown that this concentration increased the effect of respiratory viruses in AECs. ${ }^{6,8,23}$

Cell cultures were incubated at $37^{\circ} \mathrm{C}$ and cell culture homogenates and supernatants were harvested at the indicated time points. Total EGFR-p was measured at 10 and $30 \mathrm{~min}$ by enzyme-linked immunosorbent assay (ELISA) (R\&D Systems, Minneapolis, MN, USA) and nicotinamide adenine dinucleotide phosphate (NADPH) oxidase activity (Cell Technology Inc., Mountain View, CA, USA) in cell lysates was measured at $2 \mathrm{~h}$. Virus in cell culture homogenates was measured at $24 \mathrm{~h}$ by standard plaque assay. ${ }^{19}$ CXCL8, CXCL10, and IFN $-\lambda$ were measured in cell culture supernatants at $24 \mathrm{~h}$ by ELISA (eBioscience Inc.). To standardize for potential differences in productive viral infection, total protein was measured in cell culture homogenates from each experiment and results for CXCL8, CXCL10, and IFN- $\lambda$ were standardized to total protein, as seen previously. ${ }^{6,8}$ BEAS- $2 b$ cells cultured in serum-free medium, treated with chemical inhibitors, or siRNA were assessed for cytotoxicity by using a LDH assay (Roche, Indianapolis, IN, USA) and no significant differences were found previously. 6,8

IFN- $\lambda$ and IRF1 mRNA expression was assessed by quantitative RTPCR, as previously described. ${ }^{6,8}$ Total RNA was extracted using RNeasy kit (Qiagen, Valencia, CA, USA). RT-PCR was evaluated with Applied Biosystems Model 7900 sequence detector. The following primers were used: IFN- $\lambda$ (IL-29) (Forward): GGGAACCTGTGTCTGAGAACGT; IFN- $\lambda$ (IL-29) (Reverse): GAGTAGGGCTC AGCGCATAAATA; IRF1 (Forward): CTCTGAAGCTACAACAGAT GAGG; IRF1 (Reverse): CTGTAGACTCAGCCCAATATCCC. The housekeeping gene, glyceraldehyde 3-phosphate dehydrogenase, was used as an internal control.

siRNA was used to knockdown EGFR and IRF1 in BEAS-2b cells, as previously described. ${ }^{6,8,24}$ Scrambled (control) and EGFR siRNA were purchased from Santa Cruz Biotechnology (sense: CUCUGGAGGAAAAGAAAGU; antisense: ACUUUCUUUUC CUCCAGAG). Scrambled (control) and IRF1 siRNA (duplex UCCCAAGACGUGGAA GGCCAACUUU) were purchased from Invitrogen (Grand Island, NY, USA). siRNA transfection was carried out using Lipofectamine (Invitrogen) in sub-confluent cells, and $24 \mathrm{~h}$ after transfection cell cultures were infected with virus. Unless stated otherwise, Gefitinib was added at the same time as viral infection. Twenty-four hours after viral infection, cell culture homogenates were prepared to measure viral titers and cell culture supernatants were collected to measure IFN- $\lambda$. siRNA knockdown of EGFR and IRF1 was 

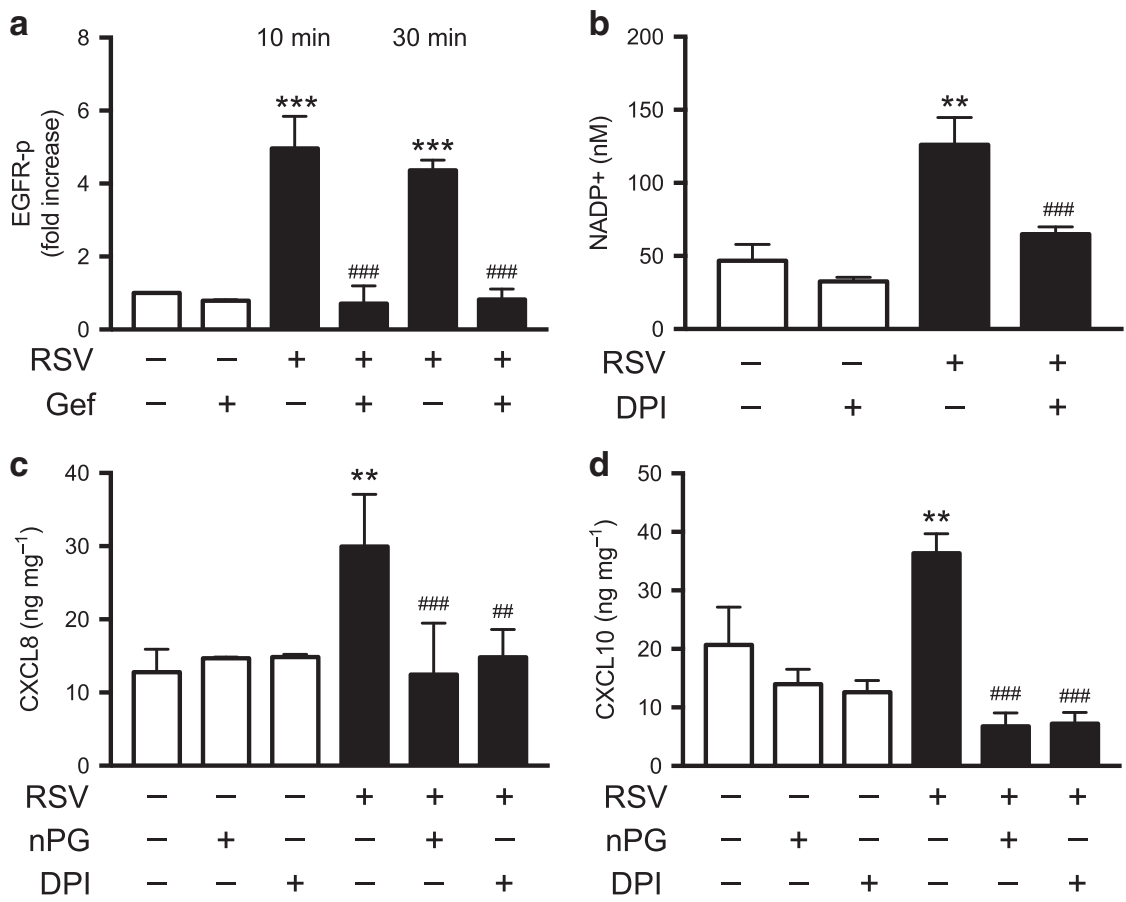

Figure 1 RSV activates EGFR and NADPH oxidases. (a) EGFR-p was measured by ELISA at 10 min (first four colums) and 30 min (last two columns) in BEAS-2b cell culture lysates. Cells were treated with serum-free medium alone (Control, empty columns), Gefitinib (10 $\mu \mathrm{m})$ alone, RSV (MOI 0.1; filled columns) alone, or with the addition of Gefitinib ( $n=3-6$ independent experiments, mean \pm s.e.m.; *** $P<0.001$ vs. control; \#\#\# $P<0.001$ vs. RSV alone). (b) NADPH activity was measured in BEAS- $2 \mathrm{~b}$ cells at $2 \mathrm{~h}$. Cells were treated with serum-free medium alone (Control, empty columns), DPI ( $3 \mu \mathrm{m})$ alone, with RSV (MOI 0.1; filled columns) alone, or with the addition of DPI ( $n=3-4$ independent experiments, mean \pm s.e.m.; ${ }^{* *} P<0.01$ vs. control; \#\#\# $P<0.001$ vs. RSV alone). (c) BEAS-2b cells were treated with serum-free medium alone (empty columns), nPG (100 $\mu \mathrm{M})$ alone, DPI ( $3 \mu \mathrm{m})$ alone, RSV (MOI 0.1; filled columns) alone, or with the addition of $\mathrm{nPG}$ and DPI, and secreted CXCL8 (IL-8) was measured by ELISA at $24 \mathrm{~h}$ ( $n=3-5$ independent experiments, mean \pm s.e.m.; ${ }^{* \star} P<0.001$ vs. control; \#\# $P<0.001$ and \#\#\# $P<0.0001$ vs. RSV alone). (d) BEAS-2b cells were treated with serum-free medium alone (empty columns), nPG $(100 \mu \mathrm{M})$ alone, DPI $(3 \mu \mathrm{m})$ alone, RSV (MOI 0.1; filled columns) alone, or with the addition of $\mathrm{nPG}$ and DPI, and secreted CXCL10 was measured by ELISA at $24 \mathrm{~h}\left(n=3-5\right.$ independent experiments, mean \pm s.e.m.; ${ }^{* *} P<0.005$ vs. control; \#\# $P<0.001$ and \#\#\# $P<0.0001$ vs. RSV alone)

confirmed by Western blot using anti-EGFR (Santa Cruz Biotechnology), and anti-IRF1 (Cell Signaling) Abs. Western blot was used to measure EGFR and IRF1. In brief, cells were lysed using RIPA buffer (Thermo Fisher Scientific, Lafayette, CO, USA) supplemented with phosphatase- and protease-inhibitors. Equivalent amounts of protein were loaded onto Mini-PROTEAN TGX 10\% gels (Bio-Rad Laboratories). After electrophoresis and blocking with TBST (Bio-Rad Laboratories) containing 5\% bovine serum albumin, blots were incubated with anti-IRF1 or anti-EGFR Ab overnight. Membranes were stripped and re-probed with mouse anti- $\beta$-actin Ab (Santa Cruz Biotechnology, sc-47778). To measure IRF1 transcriptional activity, BEAS-2b cells were transfected using TransIT-2020 Reagent (Mirus, Madison, WI, USA) with $250 \mathrm{ng}$ IRF1 luciferase reporter and the appropriate negative and positive controls (SABiosciences, Frederick, MD, USA). After $24 \mathrm{~h}$, cells were stimulated before cell lysates were prepared and IRF1 luciferase activity was assayed by Dual-Luciferase Reporter Assay System (Promega, Madison, WI, USA) according to the manufacturer's instructions.

Statistical analysis. Results are presented as both individual data points and mean \pm SE. To determine significance, two-tailed Student's $t$-test was used (GraphPad Prism version 7). $P$-values of $\leq 0.05$ were considered to be statistically significant.

\section{RESULTS}

Role for EGFR activation in RSV infection

The following studies use an AEC (BEAS-2b) line and NHBE cells to investigate the role of EGFR signaling in airway epithelial RSV infection. To investigate the role for RSV activation of EGFR in AECs, we measured total EGFR-p by ELISA following viral infection in BEAS-2b cells. RSV-stimulated EGFR-p at 10 and $30 \mathrm{~min}$, and the addition of Gefitinib, a selective EGFR tyrosine kinase inhibitor that is used clinically, decreased RSV-induced EGFR-p significantly (Figure 1a). These results confirmed that RSV activates EGFR., ${ }^{5,25}$ NADPH oxidases (Nox) produce reactive oxygen species (ROS) that are upstream of EGFR activation in AECs. ${ }^{26}$ Previously, respiratory viruses have been shown to induce Nox, ${ }^{8,27}$ and here we show that RSV activates Nox in BEAS-2b cells (Figure 1b). In addition, respiratory viruses activate EGFR to modulate CXCL8 and CXCL10 production. ${ }^{5,6,28}$ Both these molecules are inflammatory chemokines involved in hematopoietic cell recruitment (e.g., neutrophils and lymphocytes) to infected epithelium. Therefore, we stimulated BEAS-2b cells with RSV, and the addition of a ROS scavenger (nPG) and a Nox inhibitor (DPI), and measured CXCL8 and CXCL10 production. RSV alone stimulated CXCL8 (Figure 1c) and CXCL10 (Figure 1d), and the addition of nPG and DPI decreased CXCL8 (Figure 1c) and CXCL10 (Figure 1d) production significantly. Taken together, these results show that RSV activates EGFR-p, and Nox signaling is 
a

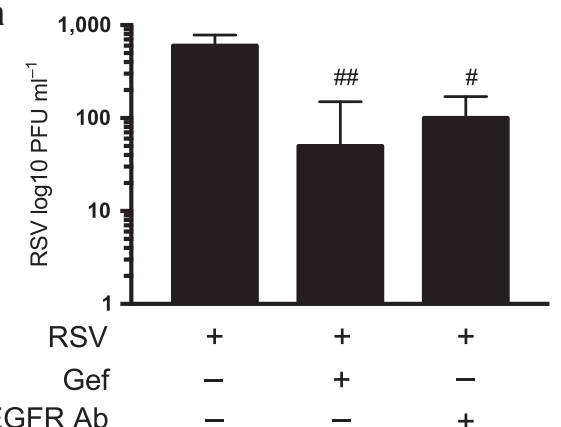

EGFR Ab

$-$

C

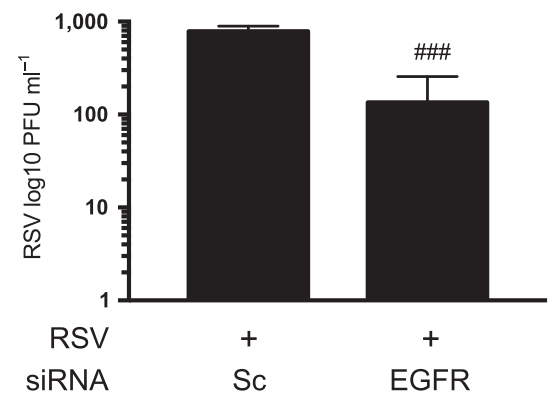

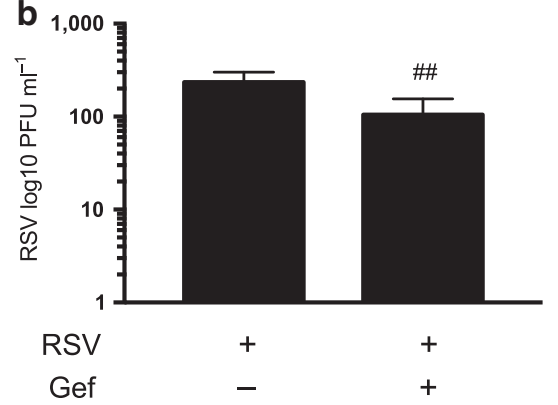

SiRNA

Sc EGFR

EGFR

$\beta$ actin

Figure 2 Role of EGFR in RSV infection. (a) BEAS-2b cells were treated with RSV alone (MOI 0.1), or with the addition of Gefitinib (10 $\mu \mathrm{m}$ ) and EGFR neutralizing $\mathrm{Ab}\left(3 \mathrm{ng} \mathrm{ml}^{-1}\right)$. After $24 \mathrm{~h}$ cell culture homogenates were collected and virus was quantified by plaque assay ( $n=4-5$ independent experiments, mean \pm s.e.m.; \# $P<0.01$ or \#\# $P<0.001$ vs. RSV alone). (b) NHBE cells were treated with RSV alone (MOI 1), or with the addition of Gefitinib $(10 \mu \mathrm{m})$. After $24 \mathrm{~h}$ cell culture homogenates were collected and virus was quantified by plaque assay $(n=4$ independent experiments, mean \pm s.e.m.; \#\# $P<0.005$ vs. RSV alone). (c) BEAS-2b cells were transfected with scrambled (Sc) or EGFR siRNA and subsequently treated with RSV (MOI 0.1). After $24 \mathrm{~h}$ cell culture homogenates were collected and virus was quantified by plaque assay $(n=4$ independent experiments, mean $\pm \mathrm{s}$.e. $\mathrm{m}$.; \#\#\# $P<0.005$ vs. RSV alone). BEAS-2b cells were transfected with EGFR siRNA and EGFR protein was assessed by western blot (representative of three independent experiments).

involved in RSV-induced CXCL8 and CXCL10 production, which implicates Nox as a shared epithelial signal in response to multiple respiratory viruses.

Epithelial inflammation augments viral infection, and we hypothesized that EGFR has a role in RSV infection. To assess the effect of EGFR inhibition on RSV infection in AECs, BEAS$2 \mathrm{~b}$ cells were infected with RSV and treated with Gefitinib, or an EGFR neutralizing $\mathrm{Ab}$ (EGFR $\mathrm{Ab}$ ), and viral titers were quantitated by plaque assay. Here, we found that EGFR inhibition suppressed RSV infection significantly (Figure 2a), which we and other investigators have observed with influenza virus, ${ }^{8,29-31}$ and rhinovirus. ${ }^{8}$ These results were confirmed in NHBE cells with RSV (Figure 2b). To confirm the specificity of chemical inhibitors, we treated BEAS-2b cells with EGFR siRNA, which suppressed EGFR protein significantly (Figure 2c, right). RSV infection of BEAS-2b cells treated with EGFR siRNA was reduced, compared with cells stimulated by RSV treated with control siRNA (Figure 2c). These results implicate an important role for EGFR in viral infection in vitro.

\section{EGFR inhibition exaggerates epithelial antiviral defense to RSV}

The mechanism for EGFR inhibition to suppress RSV infection has not been explored. Previously, our laboratory, ${ }^{6,8}$ and other investigators ${ }^{9,32,33}$ have shown that several viruses (e.g., influenza virus, rhinovirus, $\mathrm{HCV}, \mathrm{HBV}$, and Epstein-Barr virus (EBV)) activate EGFR to suppress antiviral immune responses. Therefore, to investigate the mechanism by which EGFR inhibition decreases RSV infection we evaluated the effect of EGFR signaling on endogenous airway epithelial antiviral immunity. Specifically, we focused on the potential role for EGFR to regulate epithelial IFN production, because IFNs have a critical role in innate and adaptive antiviral immunity. Recent studies have implicated IFN- $\lambda$ as the most significant IFN in epithelial responses to RSV infection, ${ }^{13,34}$ which our laboratory has previously confirmed with other respiratory viruses. ${ }^{6,8}$ However, a role for EGFR signaling to modulate IFN- $\lambda$ during RSV infection has not been investigated. To investigate the role of EGFR signaling on epithelial IFN- $\lambda$, NHBE cells infected with RSV were treated with a selective EGFR tyrosine kinase inhibitor, AG 1478. RSV induced IFN- $\lambda$ production (Figure $3 \mathbf{a}$ ), and the addition of AG 1478 augmented IFN- $\lambda$ production above the amount produced by virus alone (Figure $3 \mathbf{a}$ ) in NHBE cells. These results were confirmed in BEAS- $2 \mathrm{~b}$ cells with the addition of Gefitinib, another selective EGFR tyrosine kinase inhibitor that is used clinically (Figure 3b). AG 1295 did not increase IFN- $\lambda$ production (Figure $3 \mathbf{b}$ ). EGFR activation is a ligand-dependent process in airway epithelium, and RSV infection induces EGFR ligand release in airway epithelium ${ }^{5}$ to modulate inflammation. ${ }^{6}$ Therefore, to implicate a role 
a

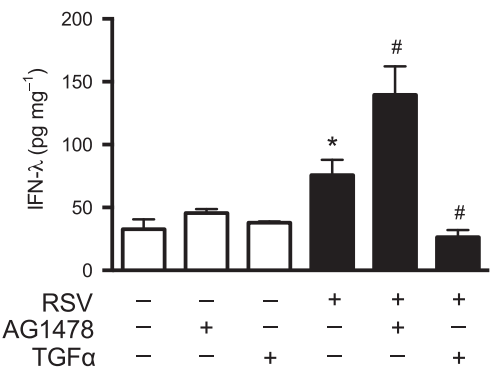

b

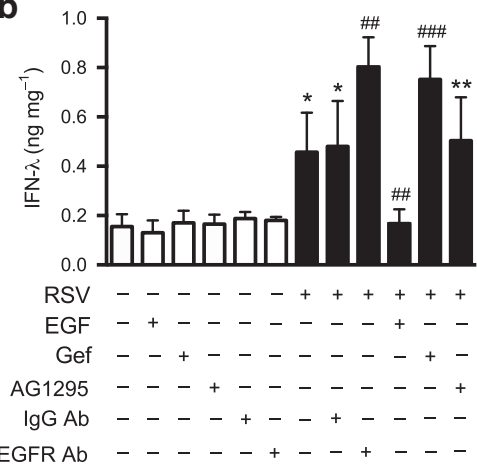

d

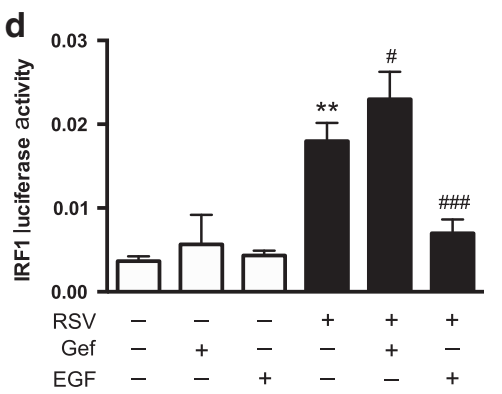

$\mathbf{f}$

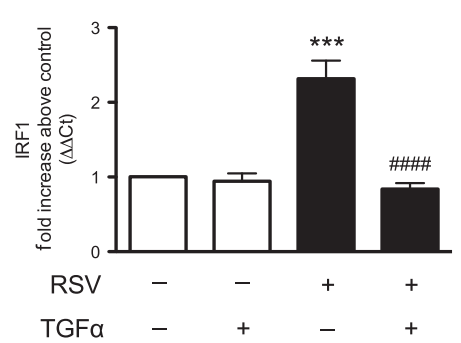

Figure 3 Effect of EGFR signaling on IRF1-dependent IFN- $\lambda$. (a) NHBE cells were treated with serum-free medium alone (empty columns), AG 1478 $(10 \mu \mathrm{M})$, TGF- $\alpha\left(10 \mathrm{ng} \mathrm{ml}^{-1}\right)$, RSV alone (MOI 1; filled colums), or with AG 1478 and TGF- $\alpha$, and secreted IFN- $\lambda$ was measured by ELISA at $24 \mathrm{~h}(n=3$ independent experiments, mean \pm s.e.m.; ${ }^{P}<0.05$ vs. control; \# $P<0.05$ vs. RSV alone). (b) BEAS-2b cells were treated with serum-free medium alone (empty columns), EGF (10 ng ml-1), Gefitinib $(10 \mu \mathrm{m})$, AG $1295(10 \mu \mathrm{m})$, an isotype-matched control Ab (IgG Ab; 3 ng ml-1), EGFR-neutralizing Ab (EGFR Ab; $3 \mathrm{ng} \mathrm{ml}-1$ ), and RSV alone (MOI 0.1; filled colums), or RSV with EGF, Gefitinib, IgG Ab, or EGFR Ab, and secreted IFN- $\lambda$ was measured by ELISA at $24 \mathrm{~h}\left(n=5\right.$ independent experiments, mean \pm s.e.m.; ${ }^{*} P<0.05$ and ${ }^{*} P<0.01$ vs. control; \#\# $P<0.01$ and \#\#\# $P<0.001$ vs. RSV alone). (c) BEAS-2b cells were treated with serum-free medium alone, or transfected with scrambled (Sc) or IRF1 siRNA for $24 \mathrm{~h}$ and treated with serum-free medium alone (empty columns), RSV (MOI 0.1 ; filled columns), or RSV and Gefitinib (10 $\mu \mathrm{m}$; 7 th column). $24 \mathrm{~h}$ after viral infection secreted IFN- $\lambda$ was measured by ELISA ( $n=6$ independent experiments, mean \pm s.e.m.; ${ }^{* \star} P<0.01$ and ${ }^{* * *} P<0.001$ vs. serum-free medium and Sc siRNA; \#\# $P<0.01$ and \#\#\# $P<0.001$ vs. Sc siRNA plus RSV). BEAS-2b cells were transfected with IRF1 siRNA and IRF1 protein was assessed by western blot (left; representative of three independent experiments). (d) BEAS-2b cells were transfected with IRF1 luciferase reporter, and after $24 \mathrm{~h}$ treated with serumfree medium alone (empty columns), Gefitinib $(10 \mu \mathrm{M})$, (EGF $\left(10 \mathrm{ng} \mathrm{ml}^{-1}\right)$, RSV alone (MOI 0.1; filled columns), and RSV plus Gefitinib and EGF for $3 \mathrm{~h}$ before luciferase activity was measured $\left(n=3-4\right.$ independent experiments in duplicate; ${ }^{* *} P<0.01$ vs. serum-free medium; \# $P<0.05$ and \#\#\# $P<0.001$ vs. RSV alone). (e) BEAS-2b cells were treated with serum-free medium alone (empty columns), TGF- $\alpha$ (10 ng ml ${ }^{-1}$ ), RSV alone (MOI 0.1 ; filled colums), and RSV plus TGF- $\alpha$ and IFN- $\lambda$ mRNA was analyzed by quantitative RT-PCR $\left(n=6\right.$ independent experiments; ${ }^{*} P<0.01$ vs. serum-free medium; \#\#\# $P<0.001$ vs. RSV alone). (f) BEAS-2b cells were treated with serum-free medium alone (empty columns), TGF- $\alpha\left(10 \mathrm{ng} \mathrm{ml}^{-1}\right), \mathrm{RSV}$ alone $(\mathrm{MOI} 0.1 ;$ filled colums), and RSV plus TGF- $\alpha$ and IRF 1 mRNA was analyzed by quantitative RT-PCR ( $n=6$ independent experiments; ${ }^{* * \star} P<0.001$ vs. serum-free medium; \#\#\# $P<0.001$ vs. RSV alone).

for RSV-dependent EGFR ligand to regulate IFN- $\lambda$ production, we treated AECs with an EGFR neutralizing antibody $(\mathrm{Ab})$ that prevented extracellular EGFR ligand-binding. In BEAS-2b cells stimulated with RSV, IFN- $\lambda$ production is increased (Figure 3b), and the addition of an EGFR-neutralizing Ab increased IFN- $\lambda$ production significantly above RSV alone (Figure 3b). In addition, EGFR neutralizing Ab exaggerates
RSV-induced IFN- $\lambda$ production to a similar level as EGFR inhibition with Gefitinib (Figure 3b).

IFN regulatory factors (IRFs) are transcription factors that have a critical role in IFN production. RSV was initially described to activate IRF1 in monocytes ${ }^{35}$ and lung epithelial cells. ${ }^{15,36}$ Subsequently, IRF1 was shown to interact with the IFN- $\lambda$ promoter, ${ }^{37}$ and we found that influenza virus, 
a

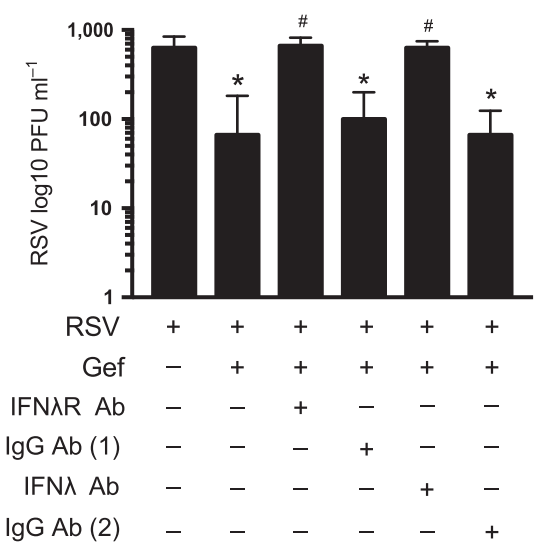

C

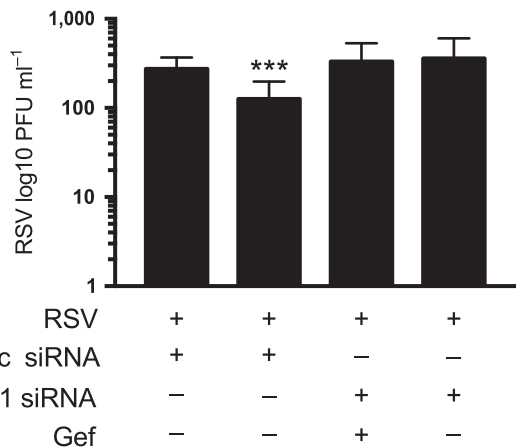

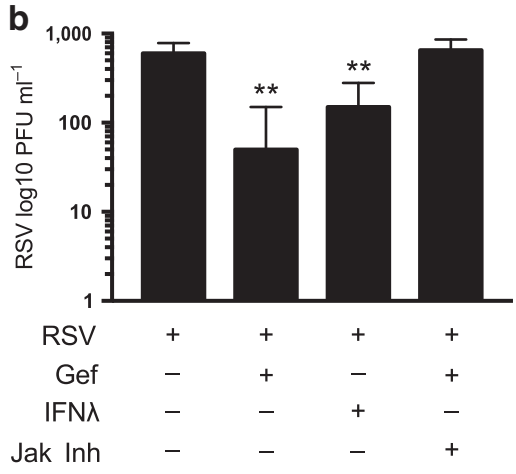

Figure 4 IFN- $\lambda$ and IRF1 are required for EGFR inhibitor-induced suppression of RSV infection. (a) BEAS-2b cells were treated with RSV (MOI 0.1) alone, with Gefitinib $(10 \mu \mathrm{m})$, and Gefitinib plus a neutralizing Ab to IFN- $\lambda$ R (IFNR Ab; columns 3), a neutralizing Ab to IFN- $\lambda$ (IFN $\lambda$ Ab; column 5 ), and isotype-matched Abs to IFNR Ab (column 4 (IgG Ab 1)) and to IFN $\lambda$ (column 6 (IgG Ab 2)) for $24 \mathrm{~h}$ and viral titers of cell culture homogenates were assessed by plaque assay ( $n=3$ independent experiments, mean \pm s.e.m.; * $P<0.05$ vs. RSV alone; $P<0.05$ vs. RSV plus Gefitinib). (b) BEAS-2b cells were treated with RSV (MOI 0.1) alone, RSV with IFN- $\lambda\left(3 \mathrm{ng} \mathrm{ml}^{-1}\right)$, Gefitinib $(10 \mu \mathrm{m})$, and Jak inhibitor $(5 \mathrm{~nm})$ for $24 \mathrm{~h}$ and viral titers of cell culture homogenates were assessed by plaque assay ( $n=4$ independent experiments, mean \pm s.e.m.; ${ }^{*} P<0.01$ vs. RSV alone; \#\# $P<0.01$ vs. RSV plus Gefitinib). (c) BEAS-2b cells were treated with serum-free medium alone, or transfected with scrambled (Sc) or IRF1 siRNA for $24 \mathrm{~h}$ and treated with RSV (MOI 0.1), or RSV plus Gefitinib (Gef, $10 \mu \mathrm{M})$. After $24 \mathrm{~h}$ cell culture homogenates were collected and viral titer was quantitated by plaque assay $(n=5$ independent experiments, mean \pm s.e.m.; ${ }^{\star \star *} P<0.005$ vs. Sc and IRF1 siRNA plus Gef).

rhinovirus, and RSV-activated IRF1 to produce IFN- $\lambda,{ }^{6}$ and subsequently other investigators have reported that IRF1 is critical for MAVS-induced IFN- $\lambda$ production. ${ }^{38,39}$ More recently, Sun Y et al., ${ }^{18}$ showed a role for IRF1 in RSVinduced IFN- $\lambda$ in a cancer cell line. To confirm a role for IRF1 in RSV-induced IFN- $\lambda$ production in AECs, we treated BEAS- $2 b$ cells with IRF1 siRNA, which suppressed IRF1 protein significantly (Figure 3c, left). RSV-induced production of IFN- $\lambda$ in BEAS- $2 b$ cells treated with IRF1 siRNA was reduced, compared with cells stimulated by RSV and treated with control siRNA (Figure 3c). In addition, IRF1 siRNA abrogated the effect of Gefitinib to exaggerate IFN- $\lambda$ protein production (Figure 3c) that was seen previously (Figure 3b). Previously, we found that IRF3 did not contribute significantly to virusinduced IFN- $\lambda$ production. ${ }^{6,8}$ Finally, RSV-induced IRF1 transcriptional activity was measured in BEAS-2b cells by IRF1 luciferase (Figure 3d). Here, RSV increased IRF1 luciferase activity (Figure 3d), and the addition of Gefitinib exaggerated this effect (Figure 3d). Therefore, these results showed that during RSV infection EGFR inhibition: (1) increased IFN- $\lambda$ production, (2) IRF1 was required for EGFR inhibition to increase IFN- $\lambda$ production, and (3) EGFR inhibition increased IRF1.
EGFR activation decreases epithelial antiviral defenses to RSV

Because we found that IRF1 is involved in RSV-induced IFN- $\lambda$ production (Figure 3c), and that EGFR inhibition was associated with increased virus-induced IFN- $\lambda$ (Figure $\mathbf{3 a}-\mathbf{c}$ ), we investigated the effect of EGFR activation to suppress these epithelial antiviral defenses. The addition of TGF- $\alpha$, a ligand that selectively binds EGFR, decreased RSV-induced IFN- $\lambda$ in NHBE cells (Figure 3a), and the addition of EGF, another selective EGFR ligand, decreased RSV-induced IFN- $\lambda$ in BEAS-2b cells (Figure $3 \mathbf{b}$ ). Next, we found that the addition of EGF also suppressed RSV-induced IRF1 transcriptional activity as measured by a luciferase assay (Figure 3d). Finally, we showed that the addition of TGF- $\alpha$ suppressed RSV-induced IFN- $\lambda$ (Figure $3 \mathbf{e}$ ) and IRF1 (Figure 3f) mRNA in BEAS-2b cells. These results showed that EGFR activation decreases airway epithelial IRF1 and IFN- $\lambda$, which implicate EGFR activation to modulate RSV-induced IRF1-dependent IFN- $\lambda$ production.

EGFR inhibition suppresses RSV via IFN- $\lambda$ and IRF1 in airway epithelium

Because EGFR inhibition elevated AEC IFN- $\lambda$ production, we examined the role of IFN- $\lambda$ in the antiviral effects of EGFR 
inhibition. Neutralizing Abs targeting IFN- $\lambda \mathrm{R}$ and virusinduced IFN- $\lambda$ were used to inhibit IFN- $\lambda$ function, either by preventing IFN- $\lambda$ binding to its receptor, or by inactivating secreted IFN- $\lambda$. BEAS- $2 b$ cells were stimulated with RSV, with the addition of Gefitinib and IFN- $\lambda$ R or IFN- $\lambda$ Abs, and viral infection was measured by plaque assay. The addition of Abs that suppressed IFN- $\lambda$ function abrogated the ability of Gefitinib to inhibit RSV infection, implicating IFN- $\lambda$ in this process (Figure 4a). The addition of IFN- $\lambda$, at a concentration similar to the amount produced by AEC in response to RSV, suppressed RSV infection to a level similar to Gefitinib (Figure 4b). Although IFN- $\lambda$ binds to a unique receptor complex (IFN- $\lambda$ R1/IL-10R2), downstream signaling is similar to other IFNs via Jak-STAT pathways. We found that the addition of a Jak1 inhibitor removed the effect of Gefitinib to suppress RSV infection (Figure $4 \mathbf{b}$ ). This implicated IFN $-\lambda$ induced Jak-STAT signaling to mediate the effect of IFN- $\lambda$ during EGFR inhibitor-induced suppression of RSV infection.

To examine the role of IRF1 in the antiviral effect of Gefitinib, BEAS- $2 b$ cells infected with RSV were treated with Gefitinib and IRF1 siRNA and compared, by plaque assay, to cells treated with Gefitinib and control siRNA. The addition of IRF1 siRNAabrogated EGFR inhibitor-induced RSV viral suppression (Figure 4c). Together, these results reveal a novel role during RSV infection of AECs for IRF1-dependent induction of IFN- $\lambda$, which is required for the antiviral effect of EGFR inhibition on RSV infection.

RSV F protein suppresses IRF1. There are two predominant glycoproteins on the surface of RSV, an attachment $(\mathrm{G})$ and fusion (F) glycoprotein. The F protein allows for RSV to fuse with cell membranes, and has been shown to also mediate viral attachment. Importantly, recent experiments have found that the $\mathrm{F}$ protein activates EGFR to induce mucin production. ${ }^{7}$ Therefore, we hypothesized that the RSV F protein would mediate the effects of EGFR to suppress IRF1. First, we measured EGFR-p after stimulating BEAS- $2 b$ cells with UVinactivated RSV (UV RSV). The addition of UV RSV activated EPGR-p (Figure 5a), which suggests that viral attachment, not replication, is required for EGFR activation. Next we found that UV RSV activated Nox activity (Figure 5b), which suggests that UV RSV activates airway epithelial Nox to produce ROS to activate EGFR-p. Finally, BEAS-2b cells were stimulated with RSV F protein to assess its effect on IRF1. Poly I:C was used to mimic viral activation of IRF1, RSV F protein was added, and IRF1 transcription was measured (Figure 5c). Here, the addition of RSV $\mathrm{F}$ protein suppressed poly I:C-induced IRF1 expression (Figure 5c), which implicates the protein to mediate the effects of EGFR-p to suppress IRF1.

\section{DISCUSSION}

RSV remains a significant human pathogen because it leads to frequent infections in children, which are associated with a subsequent risk for future asthma. Because adaptive immune responses to RSV do not provide effective antiviral immunity, infections occur throughout life. ${ }^{3}$ Thus, in adults RSV causes pneumonia and contributes to exacerbations of underlying chronic lung diseases. Importantly, despite substantial disease burden and significant efforts to identify a vaccine or antiviral therapy, there is not an approved effective therapy for RSV. Therefore, a better understanding of innate immune responses to RSV may provide novel insights into potential therapeutic targets.

AECs are the primary cell type for RSV infection, and epithelial host defense against viral infection is a battle between viral virulence and host responses. RSV infects airway epithelium by binding to recently identified receptors (e.g., nucleolin ${ }^{40}$ and CX3CR1 ${ }^{41,42}$ ), but the specific mechanism(s) for RSV entry remain to be elucidated. Viruses have evolved to hijack host responses to increase productive replication and virulence. For example, RSV contains nonstructural proteins (NS)-1 and -2 that suppress innate and adaptive immune responses against this virus. NS1 was found to inhibit type I IFN production, whereas NS2 inactivated RIG-I signaling. ${ }^{43}$ In addition, RSV NS1 protein inhibits airway epithelial IFN- $\lambda$ (lambda) production, ${ }^{14}$ and virus-induced EGFR activation may be another example to promote viral virulence. Several viruses have been shown to activate EGFR (e.g., influenza A virus, rhinovirus, RSV, cytomegalovirus, Epstein-Barr virus, vesicular stomatitis virus, and Hepatitis $B$ and $C$ viruses). In addition, our laboratory ${ }^{6,8}$ and other investigators ${ }^{9,32,33}$ have shown that several of these viruses activate EGFR to suppress antiviral immune responses. In addition, RSV-induced inflammation and mucin production are important for viral pathology. Recently, the RSV fusion (F) protein was shown to induce airway epithelial mucin production in an EGFRdependent manner, ${ }^{7}$ and prior studies showed a similar effect for RSV-induced EGFR signaling to regulate CXCL8 production. ${ }^{5,6}$ These experiments by Monick et al. ${ }^{5}$ were the first to show that RSV activates EGFR, which was subsequently confirmed by other investigators, ${ }^{7}$ and our laboratory ${ }^{6}$ (Figure 1). In addition, recent experiments suggest that the magnitude of EGFR activation may be dependent upon RSV virulence factors. ${ }^{7}$ Together, these studies have shown that RSV-induced CXCL8 and mucin production requires EGFR. ${ }^{5-7}$

In airway epithelium, EGFR activation involves an integrated signaling pathway that includes Nox-induced production of ROS that activate a metalloproteinase (e.g., TNF- $\alpha$-converting enzyme), which cleaves an EGFR proligand (e.g., TGF- $\alpha$ and EGF) that is released to bind to, and to activate EGFR. ${ }^{26,44}$ Recently, we implicated Nox as a shared epithelial signal in response to multiple respiratory viruses. ${ }^{8}$ In addition, here we found that a Nox inhibitor (DPI) and ROS scavenger (nPG) suppressed RSV-induced CXCL8 production (Figure 1). ${ }^{6}$ CXCL10 is a CXCR3 ligand, produced by airway epithelium, which contributes to NK cell and T-lymphocyte recruitment to target and kill virus-infected cells. RSV induces airway epithelial CXCL10 production, ${ }^{6,45}$ and we found that inhibition of Nox and ROS decreased RSV-induced CXCL10 (Figure 1). UV RSV and RSV F activated airway epithelial CXCL8 and CXCL10 production. ${ }^{45}$ More recently, RSV F protein was shown to activate EGFR. ${ }^{7}$ These experiments, and others, ${ }^{46}$ 
a

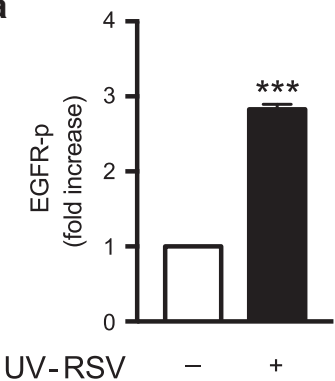

C

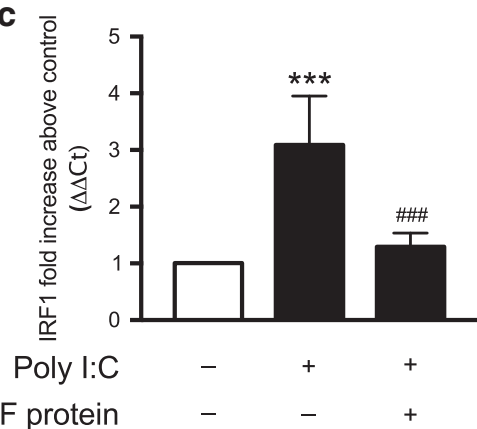

b

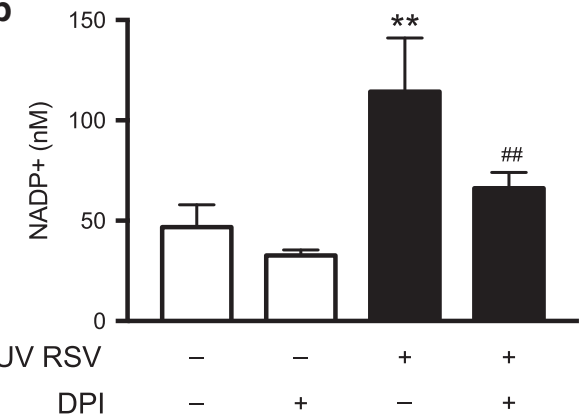

Figure 5 RSV F protein suppresses IRF1. (a) BEAS-2b cells were treated with UV-inactivated RSV (UV RSV MOI 1; filled columns) ( $n=5$ independent experiments, mean \pm s.e.m.; ${ }^{\star * *} P<0.0001$ vs. UV RSV). (b) NADPH activity was measured in BEAS-2b cells at $2 \mathrm{~h}$. Cells were treated with serum-free medium alone (Control, empty columns), DPI $(3 \mu \mathrm{M})$ alone, with UV RSV (MOI 1; filled columns) alone, or with the addition of DPI ( $n=3-6$ independent experiments, mean \pm s.e.m.; ${ }^{* *} P<0.005$ vs. control; \#\#\# $P<0.005$ vs. UV RSV alone). (c) BEAS-2b cells were treated with serum-free medium alone (empty columns), Poly I:C alone (Poly I:C $100 \mu \mathrm{g} \mathrm{ml}^{-1}$; filled colums), and Poly I:C plus RSV F protein (F protein $20 \mu \mathrm{g} \mathrm{ml}{ }^{-1}$; filled columns), and IRF 1 mRNA was analyzed by quantitative RT-PCR $\left(n=6\right.$ independent experiments; ${ }^{\star \star \star} P<0.0001$ vs. serum-free medium; \#\#\# $P<0.001$ vs. RSV alone).

highlight potential strain-specific effects of RSV for EGFR activation and inflammation in general. Here, we confirmed that UV RSV activates EGFR-p (Figure 5), and that UV RSV activates NADPH oxidase. These results, combined with prior studies that showed (1) TNF- $\alpha$-converting enzyme and EGFR ligand are required for RSV-induced CXCL8 and CXCL10, ${ }^{6}$ and (2) RSV F protein induces EGFR-p, ${ }^{7}$ suggest that RSV interacts with airway epithelium to activate EGFR via a surfacesignaling pathway. Because RSV infects airway epithelium via nucleolin ${ }^{40}$ and CX3CR $1^{41,42}$, future experiments will explore RSV receptor activation communicating with EGFR via this surface-signaling pathway (e.g., induction of ROS production that activates TNF- $\alpha$-converting enzyme to release EGFR proligand). In addition, we hypothesize that RSV F protein activates EGFR-p to mediate these effects. This suggests that targeting EGFR surface signaling may provide a mechanism to modulate RSV-induced inflammation.

In these experiments, EGFR inhibition decreased RSV titers in vitro (Figure 2), which was recently observed in vitro and in vivo. ${ }^{7}$ However, the mechanism for this effect has not been identified. We focused on the potential role for EGFR to regulate epithelial IRF1-dependent IFN- $\lambda$ production. Here we found that EGFR inhibition increased IFN- $\lambda$ and IRF1, and RSV-induced IFN- $\lambda$ production required IRF1 (Figure 3 ). Previous experiments showed that IRF3 did not contribute to IFN- $\lambda$ production. ${ }^{6,8}$ Conversely, we showed that EGFR activation decreased IRF1 and IFN- $\lambda$ (Figure 3). In addition, because the addition of RSV F protein suppressed IRF1 (Figure 5), we implicate RSV F protein to mediate the effect of EGFR activation to decrease IRF1. Overall, these results are similar to our laboratory's observations with influenza virus and rhinovirus, ${ }^{8}$ which implicates virus-induced EGFR activation as a virulence factor for multiple respiratory viruses.

In addition, we showed that EGFR inhibition required IRF1 and IFN- $\lambda$ to suppress RSV infection, and the JAK-STAT signaling pathway was implicated for this effect (Figure 4). We also found that IFN- $\lambda$ was effective to suppress RSV infection (Figure 4). Surprisingly, we are not aware of studies that address the potential therapeutic role of exogenous IFN- $\lambda$ to suppress epithelial RSV infection. ${ }^{13}$ In addition, RSV has been found to suppress IFN $-\lambda,{ }^{14}$ which suggests its important role in antiviral immunity to RSV. The mechanisms for EGFR to suppress IRF1 remain to be elucidated. Similar to EGFR inhibition, decreased ERK 1/2, a mitogen-activated protein kinase downstream from EGFR, increased virus-induced CXCL10 production, ${ }^{47}$ which is an IFN-dependent molecule. We have found that ERK inhibition increases RSV-induced IFN- $\lambda$ (data not shown). These results suggest that future experiments to investigate downstream EGFR-dependent ERK signaling will provide an opportunity to begin to elucidate the signaling intermediates between IRF1 and EGFR.

These experiments suggest a role for EGFR inhibition to increase airway epithelial innate immune antiviral responses to RSV, and potentially more broadly to other respiratory viruses. In addition, EGFR inhibition suppresses RSV-induced mucin, ${ }^{7}$ and CXCL8 production ${ }^{5,6}$ that contributes to neutrophil recruitment, which may have implications for decreasing RSV-induced asthma. ${ }^{48}$ In addition, we have shown that EGFR 

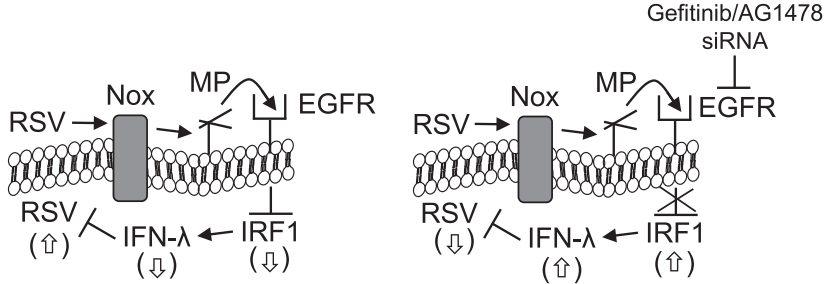

Figure 6 (Left) RSV stimulates airway epithelial NADPH oxidase (Nox), metalloproteinase (MP), and ligand-induced activation of EGFR. EGFR activation suppresses IRF1-induced IFN- $\lambda$. (Right) In the presence of EGFR inhibition (e.g., Gefitinib, AG 1478, and siRNA), IRF1-induced IFN$\lambda$ is increased, which results in decreased viral titers. Figure is adapted from Reference ${ }^{6}$.

inhibition increased respiratory virus-induced CXCL10 production, which leads to: (1) increased recruitment of lymphocytes in vivo, (2) perforin mRNA, which is found in cytotoxic T lymphocytes and NK cells, and (3) NK cell migration. ${ }^{6}$ However, targeting EGFR has potential limitations. The chronic use of an inhaled EGFR inhibitor in COPD patients was not well tolerated. ${ }^{49}$ But the potential for EGFR inhibition, ideally using an inhaled small molecule inhibitor, for a short duration after RSV infection, remains to be explored. In addition, because multiple respiratory viruses (e.g., Influenza A virus, RSV, and rhinovirus) activate EGFR to induce mucin production and neutrophilic inflammation, EGFR inhibition may have a potential benefit when viruses are identified as triggers for asthma and COPD exacerbations.

In summary, here we have examined the interaction between EGFR signaling and IRF1-induced IFN- $\lambda$ pathways in the regulation of RSV infection. RSV activated EGFR, and EGFR activation suppressed IRF1-induced IFN- $\lambda$ production (Figure 6 left). EGFR inhibition augmented IRF1 and IFN$\lambda$, which resulted in decreased viral titers (Figure 6 right). In addition, because RSV-induced Nox and ROS production leads to CXCL8 and CXCL10, we hypothesize that RSV activates EGFR via Nox and ROS upon binding to airway epithelium, although the mechanism(s) for this effect remain to be explored. In conclusion, we have uncovered a novel mechanism that RSV uses to suppress endogenous epithelial antiviral defenses (via EGFR activation), and EGFR inhibition suppresses RSV titers, which may be able to be targeted for a potential therapeutic benefit.

\section{ACKNOWLEDGMENTS}

We would like to thank Dr. Walter Finkbeiner for generously providing primary human AECs. Dr. Galen, an author on this manuscript, has a current address: department of Internal Medicine, Albert Einstein College of Medicine and Montefiore Medical Center, division of Hospital Medicine, Bronx, NY, USA. Funding: JLK was supported by NIH grant HL0923288, and is currently supported by NIH HL125897. CDC is supported by HL126094.

\section{AUTHOR CONTRIBUTIONS}

Study conception and design: JN, IU, CDC, JLK; acquisition of data: AK, $B G, I U, Y S, A M, B C, J J, A O, W L$; analysis and interpretation of data: AK, YS, JLK; drafting of manuscript: JLK; critical revision and final approval of the version to be published: AK, BG, IU, YS, AM, BC, JJ, WL, JN, CDC, JLK.

\section{DISCLOSURE}

The authors declare no conflict of interest.

c) 2018 Society for Mucosal Immunology

\section{REFERENCES}

1. Mazur, N.I. et al. Lower respiratory tract infection caused by respiratory syncytial virus: current management and new therapeutics. Lancet Respir. Med. New Haven, Connecticut, USA 3, 888-900 (2015).

2. Walsh, E.E. Respiratory syncytial virus infection in adults. Semin. Respir. Crit. Care Med. San Francisco, California, USA 32, 423-432 (2011).

3. Lambert, L., Sagfors, A.M., Openshaw, P.J. \& Culley, F.J. Immunity to RSV in early-life. Front. Immunol. New Haven, Connecticut, USA 5, 466 (2014).

4. American Academy of Pediatrics Committee on Infectious D, American Academy of Pediatrics Bronchiolitis Guidelines C. Updated guidance for palivizumab prophylaxis among infants and young children at increased risk of hospitalization for respiratory syncytial virus infection. Pediatrics 134, 415-420 (2014).

5. Monick, M.M. et al. Activation of the epidermal growth factor receptor by respiratory syncytial virus results in increased inflammation and delayed apoptosis. J. Biol. Chem. 280, 2147-2158 (2005).

6. Kalinowski, A. et al. EGFR activation suppresses respiratory virus-induced IRF1-dependent CXCL10 production. Am. J. Physiol. Lung Cell Mol. Physiol. 307, L186-L196 (2014).

7. Currier, M.G. et al. EGFR interacts with the fusion protein of respiratory syncytial virus strain 2-20 and mediates infection and mucin expression. PLoS Pathog. 12, e1005622 (2016).

8. Ueki, I.F. et al. Respiratory virus-induced EGFR activation suppresses IRF1-dependent interferon lambda and antiviral defense in airway epithelium. J. Exp. Med. 210, 1929-1936 (2013).

9. Oshiumi, H. et al. DDX60 is involved in RIG-I-dependent and independent antiviral responses, and its function is attenuated by virus-induced EGFR activation. Cell Rep. 11, 1193-1207 (2015).

10. Khaitov, M.R. etal. Respiratory virus induction of alpha-, beta- and lambdainterferons in bronchial epithelial cells and peripheral blood mononuclear cells. Allergy 64, 375-386 (2009).

11. Mordstein, M. et al. Lambda interferon renders epithelial cells of the respiratory and gastrointestinal tracts resistant to viral infections. J. Virol. 84, 5670-5677 (2010).

12. Jewell, N.A. et al. Lambda interferon is the predominant interferon induced by influenza A virus infection in vivo. J. Virol. 84, 11515-11522 (2010).

13. Okabayashi, T. et al. Type-III interferon, not type-I, is the predominant interferon induced by respiratory viruses in nasal epithelial cells. Virus Res. 160, 360-366 (2011).

14. Spann, K.M., Tran, K.C., Chi, B., Rabin, R.L. \& Collins, P.L. Suppression of the induction of alpha, beta, and lambda interferons by the NS1 and NS2 proteins of human respiratory syncytial virus in human epithelial cells and macrophages [corrected]. J. Virol. 78, 4363-4369 (2004).

15. Tsutsumi, H., Takeuchi, R., Ohsaki, M., Seki, K. \& Chiba, S. Respiratory syncytial virus infection of human respiratory epithelial cells enhances inducible nitric oxide synthase gene expression. J. Leukoc. Biol. 66, 99104 (1999).

16. Casola, A., Garofalo, R.P., Jamaluddin, M., Vlahopoulos, S. \& Brasier, A.R. Requirement of a novel upstream response element in respiratory syncytial virus-induced IL-8 gene expression. J. Immunol. 164, 59445951 (2000).

17. Liu, T., Castro, S., Brasier, A.R., Jamaluddin, M., Garofalo, R.P. \& Casola, A. Reactive oxygen species mediate virus-induced STAT activation: role of tyrosine phosphatases. J. Biol. Chem. 279, 2461-2469 (2004).

18. Sun, Y. et al. Immunostimulatory defective viral genomes from respiratory syncytial virus promote a strong innate antiviral response during infection in mice and humans. PLoS Pathog. 11, e1005122 (2015).

19. McKimm-Breschkin, J.L. A simplified plaque assay for respiratory syncytial virus-direct visualization of plaques without immunostaining. J. Virol. Methods 120, 113-117 (2004).

20. Hewson, C.A. et al. Rhinovirus induces MUC5AC in a human infection model and in vitro via NF-kappaB and EGFR pathways. Eur. Respir. J. 36, 1425-1435 (2010). 
21. Liu, K., Gualano, R.C., Hibbs, M.L., Anderson, G.P. \& Bozinovski, S. Epidermal growth factor receptor signaling to Erk $1 / 2$ and STATs control the intensity of the epithelial inflammatory responses to rhinovirus infection. $J$. Biol. Chem 283, 9977-9985 (2008).

22. Langhammer, S., Koban, R., Yue, C. \& Ellerbrok, H. Inhibition of poxvirus spreading by the anti-tumor drug Gefitinib (Iressa). Antiviral Res. 89, 64-70 (2011).

23. Subauste, M.C. \& Proud, D. Effects of tumor necrosis factor-alpha, epidermal growth factor and transforming growth factor-alpha on interleukin-8 production by, and human rhinovirus replication in, bronchial epithelial cells. Int. Immunopharmacol. 1, 1229-1234 (2001).

24. Wang, Q. et al. Role of double-stranded RNA pattern recognition receptors in rhinovirus-induced airway epithelial cell responses. J. Immunol. 183, 6989-6997 (2009).

25. Bitko, V. et al. Nonstructural proteins of respiratory syncytial virus suppress premature apoptosis by an NF-kappaB-dependent, interferon-independent mechanism and facilitate virus growth. J. Virol. 81, 1786-1795 (2007).

26. Shao, M.X. \& Nadel, J.A. Dual oxidase 1-dependent MUC5AC mucin expression in cultured human airway epithelial cells. Proc. Natl. Acad. Sci. USA 102, 767-772 (2005).

27. Fink, K., Duval, A., Martel, A., Soucy-Faulkner, A. \& Grandvaux, N. Dual role of NOX2 in respiratory syncytial virus- and sendai virus-induced activation of NF-kappaB in airway epithelial cells. J. Immunol. 180, 6911-6922 (2008).

28. Ito, Y., Correll, K., Zemans, R.L., Leslie, C.C., Murphy, R.C. \& Mason, R.J. Influenza induces IL-8 and GM-CSF secretion by human alveolar epithelial cells through HGF/c-Met and TGF-alpha/EGFR signaling. Am. J. Physiol. Lung Cell Mol. Physiol. 308, L1178-L1188 (2015).

29. de Vries, E. et al. Dissection of the influenza A virus endocytic routes reveals macropinocytosis as an alternative entry pathway. PLoS Pathog. 7, e1001329 (2011).

30. Kumar, N., Liang, Y., Parslow, T.G. \& Liang, Y. Receptor tyrosine kinase inhibitors block multiple steps of influenza a virus replication. J. Virol. 85, 2818-2827 (2011).

31. Kumar, N., Sharma, N.R., Ly, H., Parslow, T.G. \& Liang, Y. Receptor tyrosine kinase inhibitors that block replication of influenza a and other viruses. Antimicrob. Agents Chemother. 55, 5553-5559 (2011).

32. Dai, K., Huang, L., Chen, J., Yang, L. \& Gong, Z. Amphiregulin promotes the immunosuppressive activity of intrahepatic CD4 + regulatory Tcells to impair CD8 + T cell immunity against hepatitis B virus infection. Immunology 144, 506-517 (2014).

33. Wang, H.B. et al. Neuropilin 1 is an entry factor that promotes EBV infection of nasopharyngeal epithelial cells. Nat. Commun. 6, 6240 (2015).

34. Villenave, R. et al. Induction and antagonism of antiviral responses in respiratory syncytial virus-infected pediatric airway epithelium. J. Virol. 89, 12309-12318 (2015).
35. Takeuchi, R., Tsutsumi, H., Osaki, M., Sone, S., Imai, S. \& Chiba, S. Respiratory syncytial virus infection of neonatal monocytes stimulates synthesis of interferon regulatory factor 1 and interleukin-1beta (IL-1 beta)converting enzyme and secretion of IL-1 beta. J. Virol. 72, 837-840 (1998).

36. Takeuchi, R., Tsutsumi, H., Osaki, M., Haseyama, K., Mizue, N. \& Chiba, S. Respiratory syncytial virus infection of human alveolar epithelial cells enhances interferon regulatory factor 1 and interleukin-1beta-converting enzyme gene expression but does not cause apoptosis. J. Virol. 72, 44984502 (1998).

37. Siegel, R., Eskdale, J. \& Gallagher, G. Regulation of IFN-lambda1 promoter activity (IFN-lambda1/IL-29) in human airway epithelial cells. J. Immunol. 187, 5636-5644 (2011).

38. Odendall, C. et al. Diverse intracellular pathogens activate type III interferon expression from peroxisomes. Nat. Immunol. 15, 717-726 (2014).

39. Ding, S. \& Robek, M.D. Peroxisomal MAVS activates IRF1-mediated IFNlambda production. Nat. Immunol. 15, 700-701 (2014).

40. Tayyari, F., Marchant, D., Moraes, T.J., Duan, W., Mastrangelo, P. \& Hegele, R.G. Identification of nucleolin as a cellular receptor for human respiratory syncytial virus. Nat. Med. 17, 1132-1135 (2011).

41. Tripp, R.A., Jones, L.P., Haynes, L.M., Zheng, H., Murphy, P.M. \& Anderson, L.J. CX3C chemokine mimicry by respiratory syncytial virus $\mathrm{G}$ glycoprotein. Nat. Immunol. 2, 732-738 (2001).

42. Johnson, S.M. et al. Respiratory syncytial virus uses CX3CR1 as a receptor on primary human airway epithelial cultures. PLoS Pathog. 11, e1005318 (2015).

43. Meng, J., Stobart, C.C., Hotard, A.L. \& Moore, M.L. An overview of respiratory syncytial virus. PLoS Pathog. 10, e1004016 (2014).

44. Koff, J.L., Shao, M.X., Ueki, I.F. \& Nadel, J.A. Multiple TLRs activate EGFR via a signaling cascade to produce innate immune responses in airway epithelium. Am. J. Physiol. Lung Cell Mol. Physiol. 294, L1068-L1075 (2008).

45. Oshansky, C.M., Barber, J.P., Crabtree, J. \& Tripp, R.A. Respiratory syncytial virus $F$ and $G$ proteins induce interleukin 1alpha, CC, and CXC chemokine responses by normal human bronchoepithelial cells. J. Infect. Dis. 201, 1201-1207 (2010).

46. Hillyer, P. et al. Virology 505, 63-72 (2017).

47. Zaheer, R.S., Koetzler, R., Holden, N.S., Wiehler, S. \& Proud, D. Selective transcriptional down-regulation of human rhinovirus-induced production of CXCL10 from airway epithelial cells via the MEK1 pathway. J. Immunol. 182, 4854-4864 (2009).

48. Geerdink, R.J., Pillay, J., Meyaard, L. \& Bont, L. Neutrophils in respiratory syncytial virus infection: a target for asthma prevention. J. Allergy Clin. Immunol. 136, 838-847 (2015).

49. Woodruff, P.G. et al. Safety and efficacy of an inhaled epidermal growth factor receptor inhibitor (BIBW 2948 BS) in chronic obstructive pulmonary disease. Am. J. Respir. Crit. Care. Med. 181, 438-445 (2010). 